\title{
Profitable Vehicle Routing Problem with Multiple Trips: Modeling and Variable Neighborhood Descent Algorithm
}

\author{
Ahlem Chbichib ${ }^{1, *}$, Racem Mellouli ${ }^{2}$, Habib Chabchoub ${ }^{3}$ \\ ${ }^{1}$ Department of Quantitative Methods, Faculty of Economic Sciences and Management, Sfax, 3018, Tunisia \\ ${ }^{2}$ Department of computer and quantitative methods, the Higher School of Management, Sfax, 3018, Tunisia \\ ${ }^{3}$ Department of Quantitative Methods, Institute of the High Commercial Studies, Sfax, 3018, Tunisia
}

\begin{abstract}
In this paper, we tackle a new variant of the Vehicle Routing Problem (VRP) which combines two known variants namely the Profitable VRP and the VRP with Multiple Trips. The resulting problem may be called the Profitable Vehicle Routing Problem with Multiple Trips. The main purpose is to cover and solve a more complex realistic situation of the distribution transportation. The profitability concept arises when only a subset of customers can be served due to the lack of means or for insufficiency of the offer. In this case, each customer is associated to an economical profit which will be integrated to the objective function. The latter contains at hand the total collected profit minus the transportation costs. Each vehicle is allowed to perform several routes under a strict workday duration limit. This problem has a very practical interest especially for daily distribution schedules with limited vehicle fleets and short course transportation networks. We point out a new discursive approach for profits quantification which is mo re significant than those existing in the literature. We propose four equivalent mathematical formulations for the problem which are tested and compared using CPLEX solver on small-size instances. Optimal solutions are identified. For large-size instance, two constructive heuristics are proposed and enhanced using Hill Climbing and Variable Neighborhood Descent algorithm based on a specific three-arrays-based coding structure. Finally, extensive computational experiments are performed including randomly generated instances and an extended and adapted benchmark from literature showing very satisfactory results.
\end{abstract}

Keywords Profitable Vehicle Routing Problem, Multiple Trips, Mixed Integer Linear Programming, Constructive Heuristics, Hill Climbing, Variable Neighborhood Descent

\section{Introduction}

Nowadays, effective management of the supply chain is recognized as a determinant key of competitiveness and success for manufacturing and distribution organizations. The problems of routing goods from depots to consumers or between different logistical sites are very important. Drawing adequate plans may produce significant savings for many distribution systems. Therefore, the Vehicle Routing Problem (VRP) is a well-known problem studied in Operational Research. It deals with finding the optimal delivery routes configuration from one or several depots to a number of customers using a fleet of capacitated vehicles, while satisfying some constraints. The solution of a VRP is a set of minimum cost routes which fulfill the customers' requirements. In the classical version of the problem, only one depot is considered with an unlimited fleet of vehicles where each vehicle performs exactly one circuit. Several operational constraints can be considered in more practical

* Corresponding author:

ahlemchbichib@y ahoo.fr (Ahlem Chbichib)

Published online at http://journal.sapub.org/ajor

Copyright (C) 2012 Scientific \& Academic Publishing. All Rights Reserved applications of the VRP.

In this research, we focus on two variants of the VRP: the Profitable VRP and the VRP with Multiple Trips, which we combine to obtain a new variant not addressed in the literature to our knowledge and involving a new challenge in term of solving approaches. This problem which can be named the Profitable Vehicle Routing Problem with Multiple Trips (PVRPMT) calls for the determination of a set of routes for a given heterogeneous set of vehicles visiting a selective subset of customers such that: i) it is difficult, if not impossible, to visit all customers for a lack of logistical means or for insufficiency of the offer; ii) each visited customer provides a fixed profit that is recognized in the objective function to maximize. The latter is the difference between the total profit generated by the all $v$ isits and the resulting total transportation cost; iii) each vehicle may perform several routes in the same planning period but does not exceed a strict duration limit (temporal capacity); iv) for each route, the total load does not exceed the vehicle capacity (physical capacity). The motivation to study this problem come not only from its theoretical interest but also from its practical relevance. For example, the size of the vehicle fleet indicates the capital invested in logistics resources. That the reduction of this size is naturally more 
desirable, the problem counts as a strategic choice in the problem reading to guide the optimization towards reducing both capital and operating (scheduling) costs. In this situation, there might be a trade-off between higher scheduling costs and lower vehicle ownership-based costs (and eventually associated driver salaries-based costs). Otherwise, this problem is very practical where daily drivers' schedules must be achieved with a limited vehicle fleet and short-distance distribution networks. Furthermore, when we are unable to respond to all costumer requests, the problem reading should be different. A subtle selection of costumers to be served is required. It should be based on a clear and measurable criterion. It is therefore important to define a significantly manner to classify customers according to their importance, behavior and costs implications, and to translate this into a global criterion that can be optimized as the total cost associated with the transportation program. This issue of profitability will be raised when the instances are to be conceived and tested, because most companies do not have a carefully designed evaluation of each costumer's profit. The latter should not only be related to the volume of its current request but must take into account other considerations, related to the future potentials of this costumer. And the value must also be reasonably projected on a coherent scale with the transportation costs that appear in the objective function in order to ensure significant aggregation-based ass essments for the computed solutions.

The addressed problem concerns the PVRPMT as a new variant of practical VRP. Note that this kind of problems would be attractive in particular for planning mechanisms of ERP software which must integrate a miscellaneous range of situations of management and control. For a more devoted emphas is mainly to the operational context, our objective is multiple with this problem combining at hand: the maximization of the total collected profit, the minimization of the total routing costs and indirectly the maximization of the use of resources (vehicles and drivers). The issue of different mathematical models for this problem is to be addressed and used to seek solution optimality for s mall-size instances. Giving the complexity of the problem which is NP-hard, two constructive polynomial heuristics with two different greedy-based constructions will be proposed as a first approach to solve the large-size instances. Their effectiveness is tested and then enhanced, in a second solving approach, with an iterative ameliorative procedure using successive local searches with multiple and sequential neighborhood structures. For instances generation, we point out a new and quick discursive approach for profits quantification which is more significant than those existing in the literature.

The remainder of this paper is organized as follows. Section 2 reviews the related research literature. In section 3, we formally present the optimization problem PVRPMT with a detailed theoretical graph description and we introduce the corresponding proprieties and notations. Furthermore, we determine this problem complexity. In Section 4, based on two strategies of sub-tours elimination constraints, we provide four mathematical models of the problem including MILP and 0-1 ILP. Section 5 describes two constructive heuristics and an enhancement procedure based on the Variable neighborhood Descent algorithm (VND). The computational experiment is presented in Section 6 to obtain insights about the different performances of the optimizing proposed algorithms. This section begins with a new precision about the evaluation of profitability indices to be incorporated into the numerical instances' files and subsequently into the computation of the objective functions in adequacy with the measures adopted for the transportation costs. We draw conclusions and discuss future research directions in Section 7.

\section{Literature Review}

The problem we focus on is a combination of the two known variants such that: the vehicle routing problem with multiple trips or with multiple uses of vehicles (noted by VRPMT or VRPM) and the profitable vehicle routing problem (PVRP). To the best of our knowledge, these problems are studied separately in literature, excepting some special recent works[2, 3, 5], but with time windows considerations. For the latters, the concept of Multiple Trips is obtained indirectly by imposing a duration limit on each tour and not on the workday duration. Thus, the problem may look like a Capacitated VRP, where the multiple uses of vehicle is a consequence since the tours duration limit is fixed sufficiently small. So, the tours could be seen as parallel tours conducted by several vehicles. However, the integration of time windows constraint into the same problem gives the importance to the tours' order and consequently gives a special (restricted) Multiple Trips consideration. In this section, we devote two sub-sections to review the literature of the VRPMT and the PVRP.

\subsection{The Vehicle Routing Problem with Multiple Trips}

The VRPMT is an extension of the classical VRP in which the same vehicle may perform several routes in the same planning period (workday). In this case, the fleet includes a fixed number of available vehicles. However, the duration of a vehicle workday which is made of a set of successive routes does not exceed a certain limit.

This problem has a very practical importance. For example, in the home delivery of perishable goods like food, routes are of short duration and must be combined to form a complete workday[1]. Some real-world cases dealing with this kind of problem appear in applicative research publications. For instances, note the paper of Brandao et al. 1997[6] which studies the VRP in Burton's Biscuit Ltd; and the case study of the logistical activities of Santa Fe Indonesia (precisely in the office of Jakarta, a company specialized on relocation services to individuals as well as companies) which is considered in[7]. Derigs et al. 2011[8] study some real cases dealing with this problem from a consultancy company in the air cargo transportation field 
(air cargo road feeder services). Planned and ad-hoc tasks, different resources and environment constraints are taken into account, such as driving time with breaks, working time with minimum requirements in relation to the health and the safety of persons performing mobile road transport activities. These problems deals with different applications, such as: the transportation of live animals to a slaughterhouse, the transportation of the meals or the flight food from a central kitchen to the aircraft, the transportation of goods from suppliers to retailers using one or more cross-docks. More additional constraints, such as time windows, split delivery, heterogeneous fleet, are considered respectively in[9, 10, 11 and 12]. Grünert et al. 1999[13] develop a decision support system(DSS) which is designed in order to assist planners of the German postal service at the Deutsche Post AG. The main goal is to reduce the costs of the letter-mail transportation. In[14], a vehicle routing problem including multiple trips in a health care organization which operates about 240 buildings of medical offices in Southern California is treated. Finally, we mention Hernandez et al. 2009[15] which present a VRPMT applied in a context of optimizing the agronomic performance and its impact on the environment.

In the academic literature, the papers treating the VRPMT consider in general additional constraints mainly the time windows. Azi et al. 2007[1] study this problem with time windows consideration and a single vehicle's use. The extension for multi-vehicle version is tackled by Azi et al. [2, 3, 4] and Macedo et al. 2011[5]. Other works include also both multiple trips and time windows such as $[6,7,8,11,12$, $14,15,16,17,18,19$ and 20]. The overtime constraint is incorporated to the VRPMT in[14, 16, 21, 22, 23 and 24]. Heterogeneous fleet is considered with this problem in [7, 10, 12, 16 and 27]. Some VRPMT are studied by including a products compatibility constraint which forbidden to gather in a same vehicle route two or more different categories of goods [7, 8, 16, 24, and 27]. The multiple trips constraint is used in VRP with backhaul in [7 and 12], co mbined with allowed split delivery case in[12], with location problem in [25 and 26], with a planning horizon of several days in[14 and 27], with multiple depots in[28], with meal breaks during the driving time in[11]. The problem is studied in a dynamic environment in[4 and 8].

In term of solving methods, few papers develop exact approaches for the VRPMT, because of the problem's complexity, such as[1, 2, 5, 15 and 17] using MILPs, column generation with branch-and-price algorithm and network flow-based models. Note that solving the VRPMT may consist on solving a routing and packing problem. In this level, different heuristics and metaheuristics are developed. For the routing phase, we can distinguish some constructive heuristics used to obtain an initial solution, such insertion heuristics used in [3, 4, 10,14, 20, 22, 27 and 29], the Clark and Wight algorith m[24, 25 and 31], the modified Clark and Wight algorithm[25 and 31], the nearest neighborhood method[25 and 29], clustering algorithms [7, 19 and 24], the sweep-based algorithm[20, 21, 28 and
32], set covering-based approaches[7 and 32] and the petal method[32]. The initial obtained solutions are enhanced by different methods: we find the tabu search in $[6,13,14,18$, $19,20,21,23,25,27,28$ and 29], the simulated annealing in[3, 25, 26 and 31], neighborhood search based on insertion moves in[10,18,22, 24, 25, 29 and 31], on swap moves in[22, 24, 25, 26, 29 and 31], on 2-opt in[18 and 25], on adaptive memory in[21 and 30]. Other specific neighborhood search algorithms are proposed such as large neighborhood search in [3 and 4], guided neighborhood search in[8]. $\operatorname{In}[8]$, we find also a decomposition approach. In[18], a filter and fan procedure is developed. For the packing phase, some well-known heuristics for bin packing problems are used, specially the best fit decreasing in $[16,22,24,25$ and 30]. $\operatorname{In}[8]$, a g reedy packing procedure is used and a fuzzy theory-based method is proposed in[33].

The VRP with multiple trips, but no other additional constraints, is addressed through heuristics in Brandao and Mercier 1998[7], Taillard et al. 1996[23], Olivera and Viera 2007[21], Petch and Salhi 2004[22].

\subsection{The Vehicle Routing Problem with Profit}

VRP with profits are a generalization of the vehicle routing problems. Given a fixed-size fleet of vehicles, it might not be possible to serve all customers. Thus, a known profit is associated with each demand node and the customers must be chosen based on their associated profit minus the travelling cost to reach them in the solution. The idea to associate a known profit at each customer is made by Dell'A mico et al. 1995[35]. The constraint to visit all the customers is relaxed, but for each unvisited customer a given penalty has to be paid. The objective function is to find a balance between these penalties and the cost of the tour. Profits are explicitly considered both in the Vehicle Routing Problem (PVRP) and the Traveling Salesman Problem with Profits (TSPP), as stated by Feillet et al. 2005[34] in an excellent comprehensive survey. The TSPP can be formulated as a discrete bi-criteria optimization problem where the two goals are referred: maximizing the profit and minimizing the traveling cost. It is also possible to use one of the goals as the objective function and the other as a constraint. These problems can be divided into three categories according to Feillet et al. 2005[34]: a) the Profit Tour Problem (PTP), b) the Selective TSP (STSP), and c) the Prize-Collecting TSP (PCT SP).

For the first problem (PTP), the objective is to maximize the difference between the total collected profit and the traveling cost. Feillet et al. 2005[34] survey lists various modeling approaches to TSPP and exact methods as well as heuristic solution methods. Archetti et al. 2009[36] study the capacitated version of the PTP and propose exact and heuristic procedures for it. More recently, the authors [37] develop an exact approach based on a branch-and-price algorithm. A restricted master heuristic is applied at each node of the branch-and-bound tree in order to obtain primal bound values.

For the second problem which is known through three 
equivalent names in the literature: the Orienteering Problem (OP), the Selective TSP (STSP), or the Maximum Collection Problem (MCP), the objective is to maximize the collected profit such that the total traveling cost or distance does not exceed a certain upper bound. The multi-vehicles version of the OP is very similar to the VRP, except that vehicles are assumed to be with unlimited capacity and there is a time constraint on each tour. This problem is called the Team Orienteering Problem (TOP). Recently, Vansteenwegen et al. 2011[43] provides a survey of the existing OP and TOP. In this paper, the applications and the solving approaches about the two problems is reviewed listing various modeling approaches and exact as well as heuristic solution methods. In addition, many relevant variants of these problems are formally presented.

For the third problem which is named the PrizeCollecting TSP or also known as the Quota TSP concerns the determination of a tour with the minimu m total traveling cost where the collected profit or prize is greater than a lower bound.

Note that the PVRP is also a kind of PTP when considering comparing to TSPP that vehicles have physical loading capacities. In[38], The PVRP is applied in the reverse logistics where a firm aims to collect cores from its dealers. The problem is an extension of the classical multi-depots vehicle routing problem (MDVRP) in which each visit to a dealer is associated with a gross profit and an acquisition price to be paid to take the cores back. First, two mixed-integer linear programming (MILP) are presented. Then, a Tabu Search-based heuristic is proposed to solve medium and large-sized instances. In[39], the research deals with a VRP in which the total profit is to be maximized subject to market competition. The PTP in a dynamic environment is considered in[40]. In this problem, the rewards (profits) are unknown for the customers which are not yet served. Indeed, the rewards depend on competitors' prices and auctions. In another extension of the problem, the profit is associated to each edge non to vertices. The problem is then called the Profitable Arc Routing Problem (PARP). Archetti et al. 2010[41] study the capacitated undirected version (UCARPP) and develop a branch-andprice algorithm, several heuristics based on Variable Neighborhood Search (VNS) and two Tabu Search heuristics. Zachariadis et al. 2011[42] propose another local search approach for the UCARPP. Two solution neighborhoods are considered and the overall search is coordinated by the use of the promises concept.

The problem studied in this paper is a new extension which combines the two previous variants.

\section{Problem Definition and Notation}

\subsection{Problem Description and Notation}

We consider a complete undirected graph $G=(V, E)$, where $V=\{0 \ldots n\}$ is a set of vertices and $E$ is a set of edges.
Vertex 0 represents the depot and a fleet of vehicles $k=$ $\{1 \ldots m\}$ is based. Each vehicle has a limited capacity $Q$ (or $Q_{k}$ if with heterogeneous fleet) and a maximum number of trips $L$. Note that, the parameter $L$ is defined to facilitate the problem formulation and can be used as a real constraint, i.e. as a limit to be respected. However, the best value of $L$ can be experimentally identified. An edge $(i, j) \in E$ represents the possibility to travel from customer $i$ to customer $j$. A non-negative demand $q_{i}$, profit $p_{i}$, and time service $S_{i}$, are associated with each customer $i$ (with setting $p_{0}=q_{0}=0$ ). A travel time $t_{i j}$ and cost $c_{i j}$ are associated with each edge $(i, j) \in E$. Each vehicle starts and ends its tour at vertex 0 , and can visit any subset of customers with a total demand that does not exceed the used vehicle capacity $Q$. In addition, there exists a time horizon denoted by the duration limit $T_{\max }$ which establishes the duration of a workday. It is assumed that all parameters are nonnegative integers and the environment is determin istic.

This problem named the PVRPMT consists on determining a set of routes and to assign each route to one vehicle, such that the same vehicle can be used by several routes while respecting the time horizon capacity. The objective is to maximize the difference between the total collected profit and the cost of the total traveled distance. Note that the following properties:

- The optimal solution may be composed by a subset of customers.

- Each route starts and ends at the depot,

- The total customers' demand in the same route does not exceed the physical capacity of used vehicle,

- The duration of routes assigned to the same vehicle does not exceed Tmax.

- The profit associated at each customer is fixed and can be collected by any vehicle.

\subsection{Problem Complexity}

Olivera and Viera[21] proved that the VRPMT is NP-hard as well as the PVRP [34]. The studied problem represents the combination of these two NP-hard problems. This makes it also NP-hard. In addition, PVRPMT is a generalization of the classical VRP. There are instances of PVRPMT in which there exist enough vehicles in the fleet able to optimally visit during the workday all customers using one vehicle for each tour. These cases are naturally reduced to a classical VRP. As the VRP is an NP-hard[22], the PVRPMT is also NP-hard.

\section{Mathematical Models for the PVRPMT}

The design of the VRP solution stands against the presence of the sub-tours. For that, different sub-tours elimination constraints are proposed. The most classical constraint and the most used in the literature can be written in this way: 


$$
\sum_{i \in S} \sum_{j \neq i} x_{i j}^{k l} \leq|S|-1 \text { with } S \subset\{1, \ldots, n\} ; 2 \leq|S| \leq(n-1)_{(1)}
$$

This formulation is exponential and influences strongly the resolution time.

In this section, we propose four mathematical formulations for the PVRPMT. The difference between them is based on the strategy used to eliminate the sub-tours and the decision variables definitions. The different indices, parameters and decision variables are given in Table 1.

Table 1. Nomenclature

\begin{tabular}{|l|}
\hline Indices \\
\hline$i, j:$ customer index \\
$k:$ vehicle index \\
$l:$ trip index \\
$t:$ order index
\end{tabular}

\subsection{Modeling wi th 0-1 Integer Linear Programming}

We start with the idea to specify for each assigned customer his order in the trip. This idea has been applied in the scheduling problem. For each job, we determine the position of the job in the sequence. We use the visit order of customer $i$ in the trip in order to eliminate the invalid tours. Accordingly, we eliminate the subset $\mathrm{S}$ and the associated constraints. We consider the following binary decision variables:

$x_{i j}^{k l}=\left\{\begin{array}{l}1 \text { if edge }(i, j) \text { is assigned to the vehicle } k \text { during the tripl } \\ 0 \text { Otherwise }\end{array}\right.$

$y_{i t}^{k l}=\left\{\begin{array}{l}1 \text { if } i \text { is the } t^{\text {th }} \text { visited customer in the tripl } \\ \text { 0Otherwise }\end{array}\right.$

A new decision variable $y_{i t}^{k l}$ is added and informs about the visit order of customer $i$ in the trip $l$.

Based on the choice of the principal variable in the formulation, we can distinguish two different mathematical models.

- First formulation: 0-1ILP1 with $x_{i j}^{k l}$ as principal variables.

Here, the second variable $y_{i t}^{k l}$ is used just to make the connection between the edge and the vertices. The resulting formulation is the following:

$$
\operatorname{Max} \sum_{i=0}^{n} \sum_{\substack{j=0 \\ j \neq i}}^{n} \sum_{k=1}^{m} \sum_{l=1}^{L}\left(p_{i}-c_{i j}\right) x_{i j}^{k l}
$$

Subject to

$$
\begin{aligned}
& \sum_{\substack{j=0 \\
j \neq i}}^{n} x_{i j}^{k l}=\sum_{t=1}^{n+1} y_{i t}^{k l} \forall i=0, . ., n ; k=1, . ., m ; l=1, . ., L \\
& \sum_{t=1}^{n+1} \sum_{k=1}^{m} \sum_{l=1}^{L} y_{i t}^{k l} \leq 1 \quad \forall i=1, \ldots, n \\
& \sum_{i=1}^{n} y_{i t}^{k l} \leq 1 \quad \forall t=1, \ldots, n ; k=1, \ldots, m ; l=1, \ldots, L \\
& \sum_{t=1}^{n+1} y_{0 t}^{k l} \leq 1 \quad \forall k=1, . ., m ; l=1, \ldots, L \\
& \sum_{i=0}^{n} \sum_{\substack{j=0 \\
j \neq i}}^{n} q_{i} x_{i j}^{k l} \leq Q \quad \forall k=1, \ldots, m ; l=1, \ldots, L \\
& \sum_{i=0}^{n} \sum_{\substack{j=0 \\
j \neq i}}^{n} \sum_{l=1}^{L}\left(t_{i j}+S_{i}\right) x_{i j}^{k l} \leq T_{\max } \forall k=1, \ldots, m \\
& \sum_{\substack{i=0 \\
i \neq h}}^{n} x_{i h}^{k l}-\sum_{\substack{j=0 \\
j \neq h}}^{n} x_{h j}^{k l}=0 \quad \forall h=0, \ldots, n ; k=1, \ldots, m ; l=1, \ldots, L \\
& x_{i j}^{k l}+y_{i t}^{k l} \leq 1+y_{j t+1}^{k l} \forall i, j, t=0, . ., n ; k=1, . ., m ; l=1, . ., L(10) \\
& y_{j 1}^{k l}=x_{0 j}^{k l} \quad \forall j=1, \ldots, n ; k=1, \ldots, m ; l=1, \ldots, L \\
& x_{i 0}^{k l}+y_{i t}^{k l} \leq 2-\sum_{t^{\prime}=t+2}^{n+1} y_{j t^{\prime}}^{k l} \forall i, j, t=1 ., n ; k=1 ., m ; l=1 ., L \\
& M \sum_{j=1}^{n} x_{0 j}^{k l} \geq \sum_{i=0}^{n} \sum_{\substack{j=0 \\
j \neq i}}^{n} x_{i j}^{k l} \quad \forall k=1, \ldots, m ; l=1, \ldots, L \\
& x_{i j}^{k l}, y_{i t}^{k l} \in\{0,1\} \forall i, j, t=0, . ., n ; k=1, . ., m ; l=1, . ., L
\end{aligned}
$$

In this formulation, the objective is to maximize the overall collected profit minus the transportation cost. The constraints (3-5) guarantee that each customer is visited at most once. In (6), if the route $l$ exists, it should start and fin ish in the depot. (7) repres ents the capacity cons traint. The limit duration on a workday is restricted by (8). (9) represents the flow conservation constraint. The constraint (10) establish the relation between the edg $(i, j)$ and the relevant position of $i$ and $j$. (11) represents the initialization of the counter and (12) stands against the addition of a customer if the route is closed. In (13), each constructed trip should start in the depot. (14) represent the integrity constraints.

- Second formulation: 0-1 ILP2 with $y_{i t}^{k l}$ as principal 
variables

In this model, we conserve the constraints which establish the relation between $x_{i j}^{k l}$ and $y_{i t}^{k l}$ such in ILP1. When it is possible, we integrate the variable $y_{i t}^{k l}$ in the objective function and in the constraints. The objective is to test the influence of this transformation on the upper bound and to know which expression can conserve much more information if the integrity constraints are relaxed. The resulting model is as follow:

$$
\operatorname{Max} \sum_{i=1}^{n} \sum_{t=1}^{n+1} \sum_{k=1}^{m} \sum_{l=1}^{L} p_{i} y_{i t}^{k l}-\sum_{i=0}^{n} \sum_{j \neq i}^{n} \sum_{k=1}^{m} \sum_{l=1}^{L} c_{i j}^{k l} x_{i j}^{k l}
$$

subject to:

$$
\begin{aligned}
& \sum_{\substack{j=0 \\
j \neq i}}^{n} x_{i j}^{k l}=\sum_{t=1}^{n+1} y_{i t}^{k l} \forall i=0, \ldots, n ; k=1, \ldots, m ; l=1, \ldots, L \\
& \sum_{t=1}^{n+1} \sum_{k=1}^{m} \sum_{l=1}^{L} y_{i t}^{k l} \leq 1 \quad \forall i=1, \ldots, n \\
& \sum_{i=1}^{n} y_{i t}^{k l} \leq 1 \quad \forall t=1, \ldots, n ; k=1, \ldots, m ; l=1, \ldots, L \\
& \sum_{t=1}^{n+1} y_{0 t}^{k l} \leq 1 \quad \forall k=1, \ldots, m ; l=1, \ldots, L \\
& \sum_{i=1}^{n} \sum_{t=1}^{n} q_{i} y_{i t}^{k l} \leq Q \quad \forall k=1, \ldots, m ; l=1, \ldots, L \\
& \sum_{i=0}^{n} \sum_{j \neq i}^{n} \sum_{l=1}^{L} t_{i j} x_{i j}^{k l}+\sum_{i=0}^{n} \sum_{t=0}^{n} \sum_{l=1}^{L} S_{i} y_{i t}^{k l} \leq T_{\max } \forall k=1, . ., m \\
& \sum_{\substack{i=0 \\
i \neq h}}^{n} x_{i h}^{k l}-\sum_{\substack{j=0 \\
j \neq h}}^{n} x_{h j}^{k l}=0 \quad \forall h=0, \ldots, n ; k=1, \ldots, m ; l=1, \ldots, L \\
& x_{i j}^{k l}+y_{i t}^{k l} \leq 1+y_{j t+1}^{k l} \forall i, j, t=0, . ., n ; k=1, . ., m ; l=1, . ., L \\
& y_{j 1}^{k l}=x_{0 j}^{k l} \quad \forall j=1, \ldots, n ; k=1, \ldots, m ; l=1, \ldots, L \\
& x_{i 0}^{k l}+y_{i t}^{k l} \leq 2-\sum_{t^{\prime}=t+2}^{n+1} y_{j t^{\prime}}^{k l} \forall i, j, t=1 ., n ; k=1 ., m ; l=1 ., L \\
& M \sum_{j=1}^{n} x_{0 j}^{k l} \geq \sum_{i=0}^{n} \sum_{\substack{j=0 \\
j \neq i}}^{n} x_{i j}^{k l} \quad \forall k=1, \ldots, m ; l=1, \ldots, L \\
& x_{i j}^{k l}, y_{i t}^{k l} \in\{0,1\} \forall i, j, t=0, . ., n ; k=1, . ., m ; l=1, . ., L
\end{aligned}
$$

\subsection{Modeling with Mixed Integer Linear Programming}

To overcome the limitation of the classical sub-tours elimination constraint, Miller et al. 1960[44] propose a new ones which are corrected by Kara et al. 2004[45]. In this level, we propose an adaptation of these constraints to our problem. For that, we remove the decision variable $y_{i t}^{k l}$ and we define $U_{i}$. as an as sociated variable to customer $i$ used to reformu late the sub-tour elimination constraints;

Firstly, we present the ordinary model. Then an extension of this model with some proposed cuts.

- Third formulation: MILP1 (without cuts)

The formulation is the following:

$$
\begin{aligned}
& \operatorname{Max} \sum_{i=0}^{n} \sum_{\substack{j=0 \\
j \neq i}}^{n} \sum_{k=1}^{m} \sum_{l=1}^{L}\left(p_{i}-c_{i j}\right) x_{i j}^{k l} \\
& \text { Subject to } \\
& \sum_{\substack{j=0 \\
j \neq i}}^{n} \sum_{k=1}^{m} \sum_{l=1}^{L} x_{i j}^{k l} \leq 1 \quad \forall i=1, \ldots, n \\
& \sum_{i=0}^{n} \sum_{\substack{j=0 \\
j \neq i}}^{n} q_{i} x_{i j}^{k l} \leq Q \quad \forall k=1, \ldots, m ; l=1, \ldots, L \\
& \sum_{i=0}^{n} \sum_{\substack{j=0 \\
j \neq i}}^{n} \sum_{l=1}^{L}\left(t_{i j}+S_{i}\right) x_{i j}^{k l} \leq T_{\max } \quad \forall k=1, \ldots, m \\
& \sum_{\substack{i=0 \\
i \neq h}}^{n} x_{i h}^{k l}-\sum_{\substack{j=0 \\
j \neq h}}^{n} x_{h j}^{k l}=0 \forall h=0, \ldots, n ; k=1, \ldots, m ; l=1, \ldots, L \\
& q_{i} \leq U_{i} \leq Q \quad \forall i=1, \ldots, n \\
& U_{i}-U_{j}+Q \sum_{k=1}^{m} \sum_{l=1}^{L} x_{i j}^{k l}+\left(Q-q_{i}-q_{j}\right) \sum_{k=1}^{m} \sum_{l=1}^{L} x_{i j}^{k l} \leq Q-q_{j} \\
& \forall i \neq j=0, \ldots, n \\
& x_{i j}^{k l} \in\{0,1\} \forall i, j, t=0, . ., n ; k=1, . ., m ; l=1, . ., L
\end{aligned}
$$

The constraint (29) guaranties that the customer $i$ is visited at most once during the workday. (30) represents the capacity constraint. The workday duration limit is respected in (31). (32) represents the flow conservation constraint. The adaptation of Miller et al. sub-tours elimination constraints, as it is modified by Kara, for our problem is given by (33) and (34). (35) represents the integrity constraint.

- Forth formulation : MILP2 ( with cuts)

For the previous mathematical model (MILP1 without cuts), we add an optional variable $\delta^{\mathrm{kl}}$ which informs about the used vehicle. So, the correspondent constraints which establish the relation between the two decision variables will be adjo ined.

$$
\delta^{k l}=\left\{\begin{array}{l}
1 \text { if the vehicle } k \text { is used during the trip } l \\
0 \text { Otherwise }
\end{array}\right.
$$

The formulation becomes:

$$
\operatorname{Max} \sum_{i=0}^{n} \sum_{\substack{j=0 \\ j \neq i}}^{n} \sum_{k=1}^{m} \sum_{l=1}^{L}\left(p_{i}-c_{i j}\right) x_{i j}^{k l}
$$

subject to

$$
\begin{aligned}
& \sum_{\substack{j=0 \\
j \neq i}}^{n} \sum_{k=1}^{m} \sum_{l=1}^{L} x_{i j}^{k l} \leq 1 \quad \forall i=1, \ldots, n \\
& \sum_{i=0}^{n} \sum_{\substack{j=0 \\
j \neq i}}^{n} q_{i} x_{i j}^{k l} \leq Q \quad \forall k=1, \ldots, m ; l=1, \ldots, L \\
& \sum_{i=0}^{n} \sum_{\substack{j=0 \\
j \neq i}}^{n} \sum_{l=1}^{L}\left(t_{i j}+S_{i}\right) x_{i j}^{k l} \leq T_{\max } \quad \forall k=1, \ldots, m
\end{aligned}
$$




$$
\begin{aligned}
& \sum_{\substack{i=0 \\
i \neq h}}^{n} x_{i h}^{k l}-\sum_{\substack{j=0 \\
j \neq h}}^{n} x_{h j}^{k l}=0 \quad \forall h=0, \ldots, n ; k=1, \ldots, m ; l=1, \ldots, L \\
& \quad 0 \leq U_{i} \leq Q \quad \forall i=0, \ldots, n \\
& U_{i}-U_{j}+Q \sum_{k=1}^{m} \sum_{l=1}^{L} x_{i j}^{k l}+\left(Q-q_{i}-q_{j}\right) \sum_{k=1}^{m} \sum_{l=1}^{L} x_{i j}^{k l} \leq Q-q_{j} \\
& \forall k=1, . ., m ; l=1, ., L \\
& \sum_{i=1}^{n} x_{i 0}^{k l}+\sum_{j=1}^{n} x_{0 j}^{k l}=2 \delta^{k l} \quad \forall k=1, \ldots, m ; l=1, \ldots, L \\
& \delta^{k l} \leq \sum_{i=0}^{n} \sum_{j=0}^{n} x_{i j}^{k l} \quad \forall k=1, . ., m ; l=1, \ldots, L \\
& \sum_{j=0}^{n} \sum_{\substack{j=0 \\
j \neq i}}^{n} x_{h j}^{k l} \leq M \delta^{k l} \quad k=1, . ., m ; l=1, \ldots, L \\
& x_{i j}^{k l} \in\{0,1\} \quad \forall i \neq j=0, \ldots, n ; k=1, \ldots, m ; l=1, \ldots, L(46), \ldots, m ; l=1, \ldots, L \\
& \delta^{k l} \in\{0,1\} \quad \forall k=1, \ldots, m
\end{aligned}
$$

To integrate the new decision variable, three additional constraints which establish the relation between the two decision variables are adjoined. (43) means that if the route $l$ exists, it should start and fin ish in the depot. (44) guaranties that if the edge $(i, j)$ is assigned to the trip $l$, this latter should be constructed. The opposite case is presented by (45). This constraint prohibits the construction of empty route, i.e. if the route $l$ is constructed, at least one edge must be assigned at this trip.

\subsection{The Heterogeneous Fleet Case}

In this subsection, we proposed a formulation extension to solve the special case of PVRPMT with heterogeneous fleet. So, each vehicle $k$ has its own capacity $Q_{k}$. Then, some modifications on MILP2 are done. The constraint (4.3) is replaced by (5.3). The constraints (4.6) and (4.7) are replaced respectively by (5.6) and (5.7):

$$
\begin{gathered}
\sum_{i=0}^{n} \sum_{\substack{j=0 \\
j \neq i}}^{n} q_{i} x_{i j}^{k l} \leq Q_{k} \quad \forall k=1, \ldots, m ; l=1, \ldots, L \\
0 \leq U_{i} \leq Q_{k} \quad \forall i=0, . ., n \\
U_{i}-U_{j}+\sum_{k}^{m} \sum_{l}^{L}\left(Q_{k} x_{i j}^{k l}+\left(Q_{k}-q_{i}-q_{j}\right) x_{i j}^{k l}\right) \\
\leq \sum_{k}^{m} \sum_{l}^{L}\left(Q_{k}-q_{i}\right)\left(x_{i j}^{k l}+x_{j i}^{k l}\right) \forall k=1, \ldots, m ; l=1, \ldots, L
\end{gathered}
$$

\section{Solving Algorithms}

The present section is devoted to the description of the main steps of the implemented algorithms. We start by explaining the solution coding scheme. Then, constructive heuristics used to build initial feasible solutions to the problem are described. Finally, improvement procedures based on Hill Climb ing and Variable Neighborhood Descent (VND) are proposed.

\subsection{Solution Coding Scheme}

To further speed up the computation, we use tree-array data structure to represent a solution. The information concerning the customer is stored into the first array $(V 1[j])$. So, its size is equal to the number of customers $n$ (we eliminate the deposit from the solution representation). First,

\begin{tabular}{|c|c|}
\hline$j$ & $1,2,3 \ldots \ldots \ldots \ldots \ldots \ldots \ldots \ldots \ldots \ldots \ldots \ldots \ldots \ldots$ \\
\hline$V 1[j]$ & 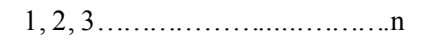 \\
\hline$V 2[j]$ & Binary vector $(0,0,1,0,0,1,0,0)$, \\
\hline$V 3[j]$ & $1,2,3 \ldots \ldots \ldots \ldots \ldots,-1,-1,-1,-1,-1$ \\
\hline
\end{tabular}
the visited customers are stored adjacently in the order of visit. Then, the unvisited customers are inserted in the end of the array. It is important to know the start and the end of each trip. This information is done by the second array ( $V 2[j])$. This latter is a binary vector. Finally, the third array $(V 3[j])$ informs about the index of the used vehicle to visit the correspondent customer. Using this structure, the lecture of the solution is clear and easy, and changes can be performed very quickly and in a constant time. The solution is coded as follows:

Let the matrix $\mathrm{V}[i][j]$ with $i \in\{1,2,3\}$ and $j \in\{1,2, \ldots \mathrm{n}\}$ be the following:

$V 1[j]=$ Customer index

$V 2[j]=\left\{\begin{array}{l}1 \text { if } j \text { is the last visited customer in the current trip } \\ 0 \text { Otherwise }\end{array}\right.$

$V 3[j]=\left\{\begin{array}{l}\text { Index of the used vehicle to visit the customer } j \\ -1 \text { if the customer } j \text { is unvisited }\end{array}\right.$

Figure 1 represents an illustrative example with 5 short routes, 18 customers and 3 vehic les and table 4 represents the relative solution code.

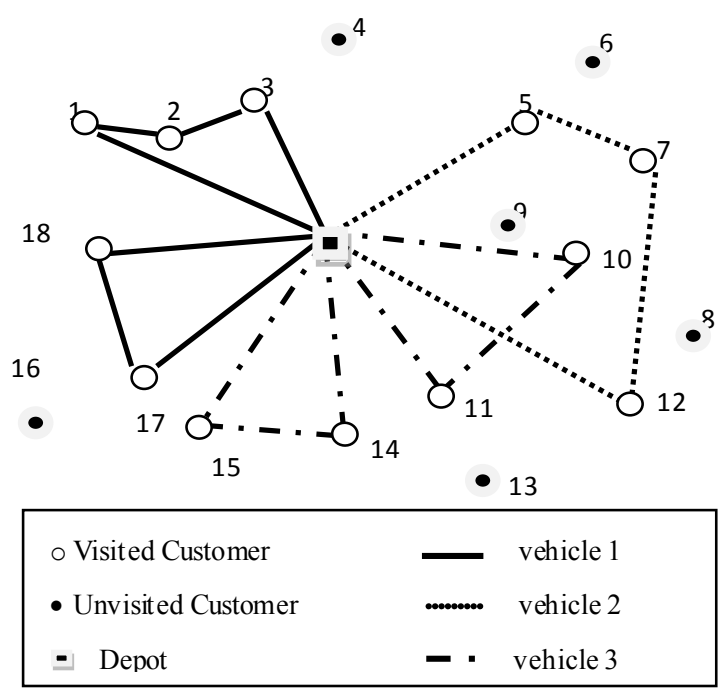

Figure 1. Illustrative Example 
Table 2. Coding Solution of the illustrative example

\begin{tabular}{|c|c|c|c|c|c|c|c|c|c|c|}
\hline $\mathrm{j}$ & 1 & 2 & 3 & 4 & 5 & 6 & 7 & 8 & 9 & 10 \\
\hline $\mathrm{V} 1[\mathrm{j}]$ & 1 & 2 & 3 & 5 & 7 & 12 & 10 & 11 & 14 & 15 \\
\hline $\mathrm{V} 2[\mathrm{j}]$ & 0 & 0 & 1 & 0 & 0 & 1 & 0 & 1 & 0 & 1 \\
\hline $\mathrm{V} 3[\mathrm{j}]$ & 1 & 1 & 1 & 2 & 2 & 2 & 3 & 3 & 3 & 3 \\
\hline
\end{tabular}

\begin{tabular}{|c|c|c|c|c|c|c|c|c|}
\hline $\mathrm{j}$ & 11 & 12 & 13 & 14 & 15 & 16 & 17 & 18 \\
\hline $\mathrm{V} 1[\mathrm{j}]$ & 17 & 18 & 4 & 6 & 8 & 9 & 13 & 16 \\
\hline $\mathrm{V} 2[\mathrm{j}]$ & 0 & 1 & 0 & 0 & 0 & 0 & 0 & 0 \\
\hline $\mathrm{V} 3[\mathrm{j}]$ & 1 & 1 & -1 & -1 & -1 & -1 & -1 & -1 \\
\hline
\end{tabular}

\subsection{Constructive Heuristics for the PVRPMT}

The goal of this subsection is to construct good feasible solutions for the problem. We propose two greedy constructive heuristics. These heuristics use some local optima lities in certains steps of the algorithm.

\subsubsection{Heuristic $\mathrm{H} 1$}

The insertion heuristic is used to build an initial feasible solution. In every iteration, the procedure evaluates all feasible insertions of unvisited nodes and selects the node representing the best insertion. An insertion is evaluated with the following criterion. Let $i$ be some node in a tour and let $j$ be a node candidate for the insertion. Let $C h g_{a c t}$ and $T m_{a c t}$ denote the actual charge and the traveled time for the current used vehicle respectively. The insertion of $j$ is feasible if the capacity constraint and the constraint of time duration are verified (i.e. (51) and (52) are verified):

$$
\begin{gathered}
\operatorname{chg}_{a c t}+q_{j} \leq Q_{k} \\
\mathrm{Tm}_{a c t}+t_{i j}+S_{j}+t_{j 0} \leq T \max
\end{gathered}
$$

Then, the best insertion is determined by the pair $(i, j)$ for which $p_{i j}$ is maximum:

$$
P_{i j}=p_{j}-c_{i j}
$$

Where $P i j$ the profit generates by the insertion of the customer $j$ after the customer $i, p_{j}$ the profit associated with customer $j$ and $c_{i j}$ the cost transportation between $i$ and $j$. The route is considered as completed if the vehicle can not receive any mo re other costumers due to its physical capacity. The vehicle returns to the depot and a new trip starts with the same used vehicle in the last iteration. The vehicle is changed only if the daily time horizon $T_{\max }$ will be violated by the addition of a new trip with the same vehicle. In this case, the next trip is done by the next vehicle (see Figure 2).

Remarq 1: in the case of a heterogeneous fleet, the vehicles are sorted in the decreasing order of their physical capacities. This order is used as a priority rule for the vehicle choices.

\subsubsection{Heuristic $\mathrm{H} 2$}

This heuristic $\mathrm{H} 2$ is almost identical with heuristic H1 with the following basic difference : to construct the trip, we use the same previous procedure, but the used vehicle is not necessary kept in prior for the next trip. At each iteration, we choose the vehicle which has the longest remaining time service by breaking ties with the largest capacity order (see Figure 3).

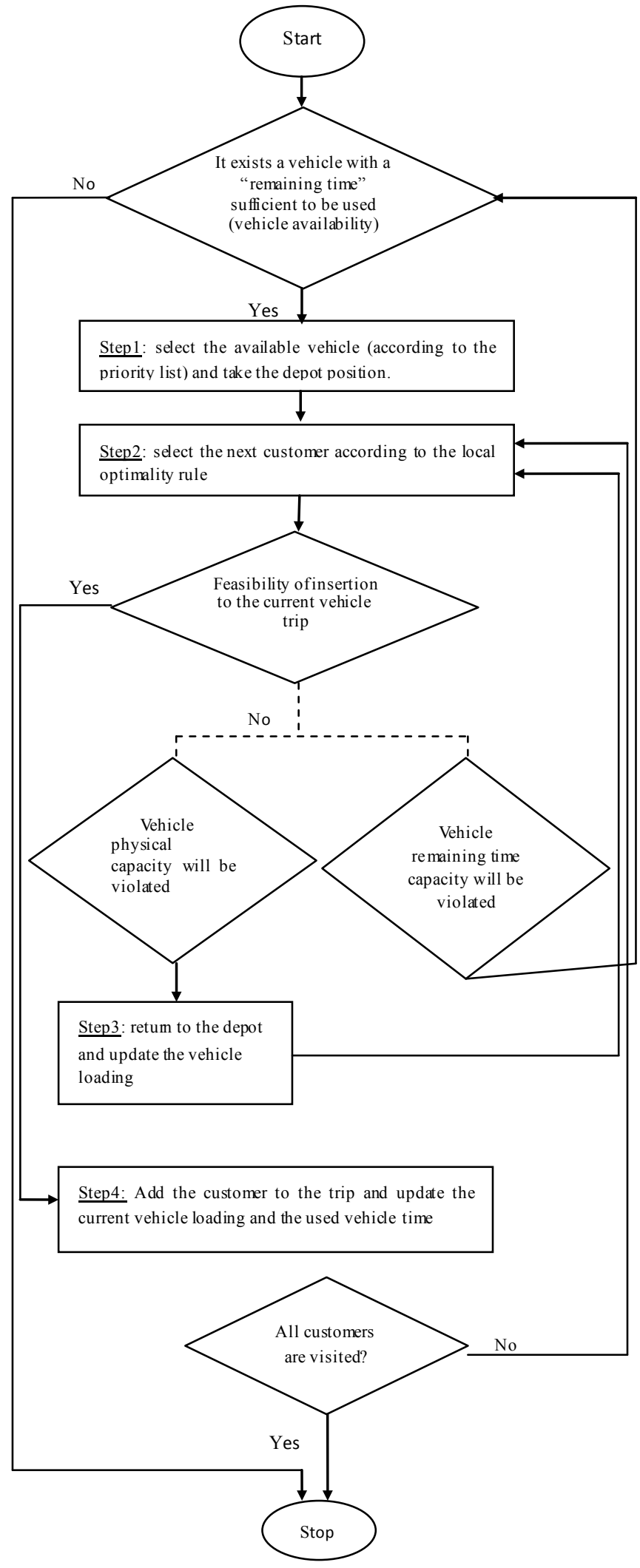

Figure 2. Constructive Heuristic 1 


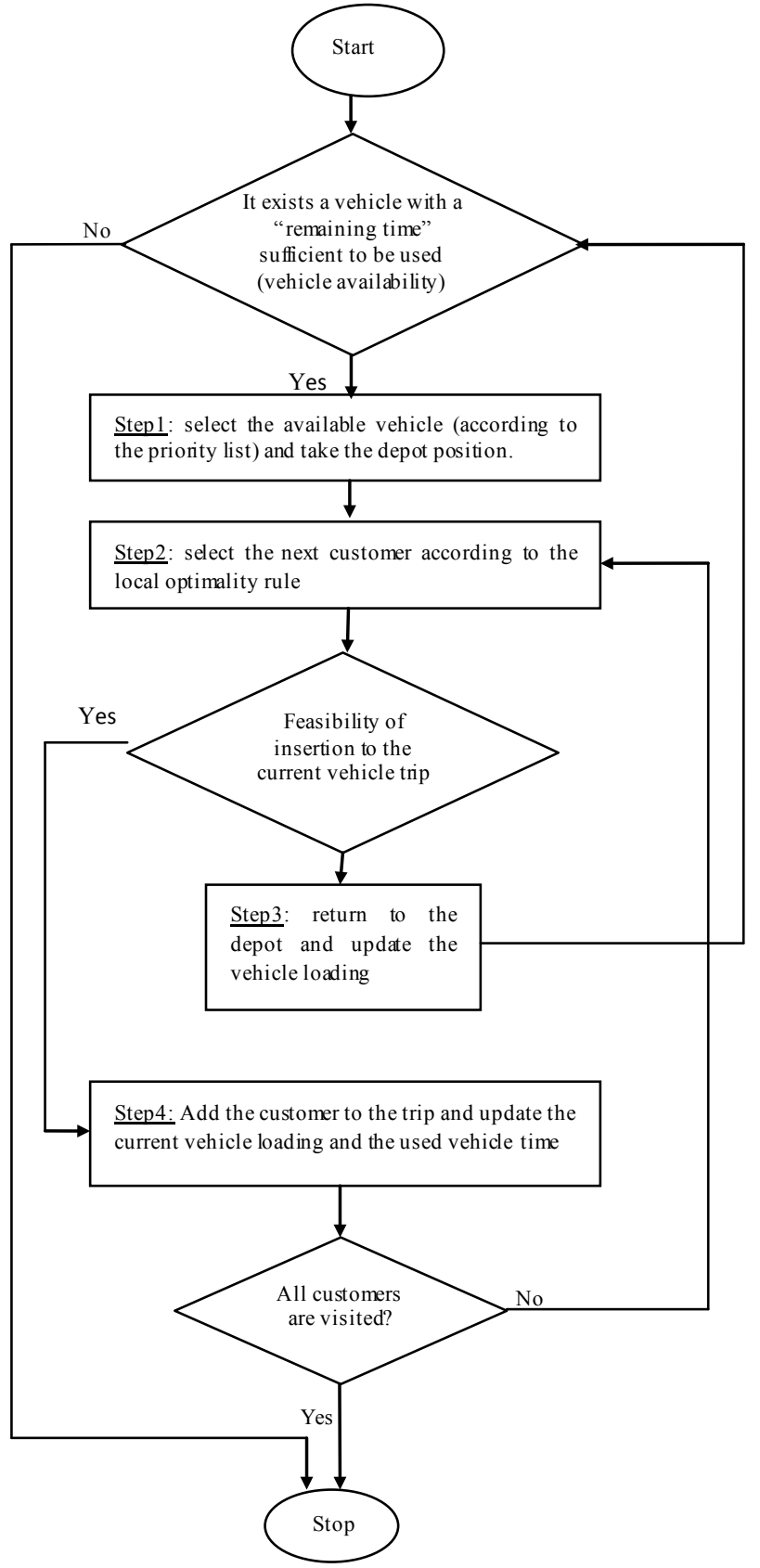

Figure 3. Constructive Heuristic 2

Remarq 2: in the case of heterogeneous fleet, we use the same priority rule for choices used in $\mathrm{H} 1$.

\subsection{Improve ment Sche me}

For the PVRPMT, it is essential to have a neighborhood that changes the visit combinations for customers. Three kinds of moves including insertion move, swapping move and Cross exchange operator move are used to define a set of neighborhoods that allow the exploration of increasingly distant solutions from the incumbent to overcome local optimality and strive for global optimality.

Our improvement phase consists on first developping three Hill Climbing algorithm ( $\mathrm{HCi}, \mathrm{HCs}, \mathrm{HCc})$ using the three neighborhood structures, then we test the influence of combining the three last algorithms in a Variable Neighborhood Descent procedure.

\subsubsection{Hill Climbing Procedure}

The local search algorith ms show its performance to solve various variants of routing problems. Here we use the Hill Climbing heuristic which belongs to the family of local search methods which often built on neighborhood moves that make small changes to the current solution.

The insert move consists of removing one customer from a current position $j$ (origin position) and putting him into another new position $k$. The destination route (i.e. the route that contains the new position) can be an existing route or a new one. To take into account all the possible moves, a new decision variable $\alpha$ is defined.

$$
\alpha=\left\{\begin{array}{l}
\text { prec }: j \text { will be inserted in thelast trip } \\
\text { suiv }: j \text { will be inserted in the next trip }
\end{array}\right.
$$

All depends on the positions of $j$ and $k$ and the variable $\delta$, we can distinguish different possible moves.

* If $\mathrm{j}=\mathrm{k}$, three scenarios can be distingushed:

- $k$ is the position of the first visited customer in the current trip (i.e. $V 2[k-1]=1$ )

- if $\alpha=$ prec the customer $i$ will be inserted in the last trip (i.e. $V 2[k-1]=0, V 2[k]=1$ and $V 3[k]=V 2[k-1]$ )

- if $\alpha=$ suiv no change in the initial solution

- $k$ is the last visited customer in the current trip $(V 2[k]=1)$

- if $\alpha=$ prec no change in the initial solution

- if $\alpha=$ suiv the customer $i$ will be inserted in the next trip (i.e. $V 2[k-1]=1, V 2[k]=0$ and $V 3[k]=V 3[k+1]$ ).

- Otherwise : there is no change

$*$ If $\mathrm{j} \neq \mathrm{k}$, accord ing to the value of $\mathrm{V} 2[\mathrm{k}]$ two different cases can appear :

- If, in the initial solution, $(V 2[k]=1)$ : two insert moves are presented

- $\alpha=$ prec: $i$ will be the last visited customer who belongs to the current trip $(V 2[i]=1$ and $(V 3[i]=V 3[k])$

$-\alpha=$ suiv : $i$ will be the first visited customer in the next trip $(V 2[i]=0$ and $(V 3[i]=V 3[k+1])$.

- if $(V 2[k]=0)$, the new solution will be presented as follows: $V 1[k]=V 1[j], V 2[k]=0$ and $V 3[k]=V 3[k-1]$.

the swap move consists on exchanging two customers. In the new solution just the position of the two selected customers will be exchanged $(V 1[k]=V 1[j])$.

The cross-exchange operator consists on interchanging non-consecutive customer segments between the same or two different routes with the restriction that the orientation of them be maintained.

Remarq 3: Note that, to elliminate the neglected move, for the two first neighborhoods (insertion and swap), at least one from the two selected customers should represent a visited customer in the initail solution. For the cross-exchange operator, the two selected customers should be visited in the initail solution.

\subsubsection{Variable Neighborhood Descent Algorithm}

The second idea is to test the influence of combining the 
three neighborhood structures in the same algorithm. So, we develop a variable neighborhood decent algorith $\mathrm{m}$ (VND).

The VND is the simplest variant of the variable neighborhood search heuristic (VNS) which performs several descents with different neighborhoods until a local optimum for all considered neighborhoods is reached. Let $\mathrm{N}_{1}, \mathrm{~N}_{2}, \ldots, \mathrm{N}_{\mathrm{k}}$ denote a set of $\mathrm{K}$ neighborhood structures (i.e., $\mathrm{N}_{\mathrm{i}}(\mathrm{S})$ contains the solution that can be obtained by performing a local change on $\mathrm{s}$ according to the $\mathrm{i}^{\text {th }}$ type).

The VND works as follows:

Step 1. Choose an initial solution $s$ in $S$.

Step 2. Set $i:=1$ and $s_{\text {best }}:=s$.

Step 3. Perform a descent form $s$, using neighborhood

$N_{i}$. Let $s^{\prime}$ be the resulting solution. If $f\left(s^{\prime}\right)<f(s)$ then set $s:=s^{\prime}$. Set $i:=i+1$. If $i \leq K$ then repeat Step 3 .

Step 4. If $f(s)<f\left(s_{\text {best }}\right)$ then go to Step 2; else stop.[46]

\section{Computational Results}

In the following, obtained results concernening the performance of the different proposed mothods are reported. First, the test instances construction, the parameters choice and the profit quantification are presented. Then, the performance of the equivalent presented models are tested and compared. Finally, the last subsection is devoted to the heuristics procedures performance (contructive, Hill Climbing, VND). The MILPs and solving algorithms was tested on a Intel Core 2 Duo CPU 2.20 GHZ and 4.00 Go RAM. The codes are written in $\mathrm{C}++$, using CPLEX lib rairies for the first part. All the algorithms were stopped before a computational time of one hour at atmost.

\subsection{Test Instances and Parameters Choice}

The tests will be applied first on our own benchmark devoted for small-size instances and then on the benchmarks of Taillard et al. 1996[23] taken from the VRP library with adaptations and some extended data. In our benchmark, twenty small-size instances are generated randomly. A certain setting is used to obtain interesting instance values according to the practice and real-case situations. For each instance, we indicate the number of vertices $n$ which ranges from 6 to 20. The customers are randomly distributed in two-dimension area, and the depot is set at point $(0,0)$. the fleet of vehicles $m$ accounts for 2 or 3 vehicles. The vehicle physical capacity limit $Q$ ranges from 1000 to $3000 \mathrm{kgs}$. The horizon time limit $T_{\max }$ for all the s mall size instances is equal to 480 minutes (i.e. 8 hours of workday).

Note that in the litterature according to[36], the profit $p_{i}$ of customer $i$ depends on the three parameters namely: cons, $h$ and the customer's demand $q_{i}$, where $h$ is a random ratio number uniformly generated in the interval $[0,1]$ and cons is a constant factor that measures the profit according to a sale turnover. $\operatorname{In}[36]$, the authers consider $p_{i}=($ cons $+h) q i$ and suppose that cons $=0.5$ indifferently with the level of greatness of the other values used for the instances data. This implies a lack of guarantee of a necessary coherence between the different values while the choices of the units and the level of greatness of the four used data are unambiguous: costomers' demands $q_{i}$, distances $d_{i j}$, transportation times tij and transportation $\cos$ ts $c_{i j}$.

In our opinion, it is important to obtain meaningful and significant proportions of the profits according to the transportation costs. In our case, they are assimilated to $d_{i j}$. Concerning the profit calculation, we take as reference a general realistic model where the logistical cost represents between 5 and 10 per cent of the sale turnover, and the gross benefit may represent between 20 and 50 per cent of the sale turnover. So, it is possible to have a g lobal profit which ranges almost between 3 and 5 times the gobal transportation cost. The distribution of this global profit on customers may respect the demand quantity $q_{i}$ of each customer $i$. Let $q_{\max }$ and $q_{\text {min }}$ be respectively the greatest and the smallest value of all demand quantities. $q_{\max }$ and $q_{\min }$ can be associated respectively to $\alpha_{\max }=5$ and $\alpha_{\min }=3$ factors. We use a projetion to calculate the factor $\alpha_{i}$ of the customer $i$ according to his demand $q_{i}$.

In order to estimate the transportation cost, we calculate firstly the average distance, identify and $\alpha_{i}$ and $p_{i}$ such as:

$$
\begin{gathered}
\bar{d}=\frac{\sum_{i} \sum_{j \neq i} d_{i j}}{(n-1)^{2}} \\
\frac{\alpha_{i}-\alpha_{\min }}{q_{i}-q_{\min }}=\frac{\alpha_{\max }-\alpha_{\min }}{q_{\max }-q_{\min }} \\
p_{i}=\alpha_{i}(1+h) \bar{d} .
\end{gathered}
$$

Note that $t_{i j}$ are also assimilated to $d_{i j}$ and the level of greatness of the vertices coordinates values is chosen adequately to obtain significant $t_{i j}$ values suitable with $T_{\text {max }}$.

In addition, we point out that $L$, which is used in MILP formulations, represents the total number of trips which can be made by a vehicle during $T_{\max }$. Its value can be estimated as :

$$
\min \left\{\left\lceil\frac{\sum Q_{k}}{\min Q_{k}}\right\rceil ;\left\lceil\frac{\sum Q_{k}}{\min Q_{k}}\right\rceil \frac{T_{\max }}{n \bar{d}}\right\}
$$

For the original instances of Taillard et al. 1996, we add all previous adaptations and extensions, as well as the service time $S_{i}$ and the maximum number of trip which can be assigned at each vehicle $L$.

\subsection{Mathematical Models Experimentations}

In order to test the proposed models, we used the commercial solver CEPLEX 10.0. of ILOG ${ }^{\circledR}$.

First, the four mathematical models are teted on the small size instances and the results are reported in Table 3.The optimal solution obtained by CPLEX is indicated under column $f$. The " " means that we cannot obtain the optimal solution before the time limit. The column $U B$ represents the solution of the linear relaxed problem (the upper bound). In addition, (\%) represents the gap between the optimal 
solution and the relevant upper bound. $(\%)=(f-U B) / U B \times$ 100.

We observe on Table 3 that the optimal value can be obtained within the time limit just for small size instances for the four mathematical models. For the instances where the number of vertices is superior to 17, the optimal solution cannot be determined. We can show also that computationnel time grow strongly with the increase of instance size. MILP 2 is able to solve more instances than the other proosed models and it clearly outperforms them in terms of computing time.

In Table 4, we try to compare the performance of the four mathematical models by confronting the obtained upper bounds. We choose as reference the upper bound of the first mathe matical model (UB1) and we calculate the deviation of the different upper bounds in comparison with the first. $(\% *) w=(U B w-U B 1) / U B 1 \times 100$.

Different remarks can be taken into account. First by comparing the two strategies, the $0-1 I L P$ and the MILP, it is very clear that the upper bounds obtained by the mixed integer programming are better than those obtained by the $0-1$ integer programming for all the tested instances. For the $0-1$ ILP, the choice of the principal variable has not great influences on the upper bounds quality (for the majority of the tested instances the upper bound obtained by the two models are equal) but strongly affects the number of iterations. For the $M I L P$, the upper bounds of the mathematical models with cuts are better than those without cuts. That shows the efficiency of these cuts. MILP2 is able to solve more instances than the other mathematical models and outperforms them in terms of computing time and the upper bound quality. So, we can judge that the additional constraints represent valid cuts for our model.

\subsection{Heuristics Procedure Perfor mance}

To test and compare the performance of our heuristics, we compute the obtained gaps of the obtained solutions comparing to the linear upper bounds. These gaps are given: first for the two constructive heuristics bounds, second for the three Hill Climbing based solutions, and finally for enhancement by using the Variable Neighborhood Descent algorith $\mathrm{m}$. Gaps are also computed comparing to the optimal solution for the small-size instances. Then, to know the contribution generated by the VND algorithm, we calculate the gap between the initial solution given by the constructive heuristics and the lower bound. As it is previously mentioned, MILP2 represents the best formulation of our problem. So, the lower bound given by our heuristic will be compared with the upper bound obtained with this formulation.
The test includes our twenty instances generated randomly with adequate settings and forty adapted instances selected fro $m$ the bench marks of Taillard et al. 1996[23]. The results are shown in table 5 and 6 .

The column $f$ and $U B$ represent respectively the optimal solution and the upper obtained by the linear relaxation of MILP2. The initial solution of our constructive algorithms (Constructive heuristic 1 and constructive heuristic 2) is given in column $H 1$ and $H 2$. The Hill Climb ing improvement tested for the three neighborhood structures by the insertion move, the swap move and the cross-exchange are showed in the column $\mathrm{HCi}, \mathrm{HCs}$ and $\mathrm{HCc}$ respectively. The column $V N D$ gives the lower bound obtained with the Variable Neighborhoods Decent algorithm. The column gap and gap* represent respectively the gap between the lower bound (the $V N D$ solution) and the optimal solution and the upper upper respectively. The column gap ${ }^{* *}$ calculates the enhancement generated by the $V N D$ algorithm by mesuring the gap between the $V N D$ and the initial solution.

Fro $m$ the first observation and by comparing the three Hill Climbing shemes, we can see that these procedures give several acceptable results for the most tested instances. Howerever, some of them still require further enhancement. On the 12 instances for which the optimal solution is determined, the $V N D$ algorithm is managed to find the optimal solution in $50 \%$ of cases. For the others, the gap is generally tiny, except for few exceptional instances, where it is quite signeficant reaching for the worst $33 \%$. For the large size instances, this gap (gap*) becomes important and grows continuously because it is computed compared to the linear bound and not to optima. The VND algorithm enhances clearly the initial solution for the total of the tested instances. But, for few instances (i.e. instances 7 and 8) the improvement is small and the $V N D$ converges quickly to local maxima.

To crown all, we can conclude that the MILP2 (with cuts) represents the best formulation of our problem. The two constructive heuristics and the enhancement procedure based on the Hill Climbing and the $V N D$ algorithm produce acceptable solutions close to the optimal ones for s mall size instances. For the big size instances, the obtained solutions are the best till now. In a future works, we think about enhancing more and more the upper bounds with some polyhedral techniques to have a clearer idea about the performance of our proposed methods for the large size instances. To escape from a current local optimum, we should think to add, in a third solving phase, some perturbation moves to strengthen the search diversification in our algorithms. Best performances could be deduced in future works after some enhancements. 


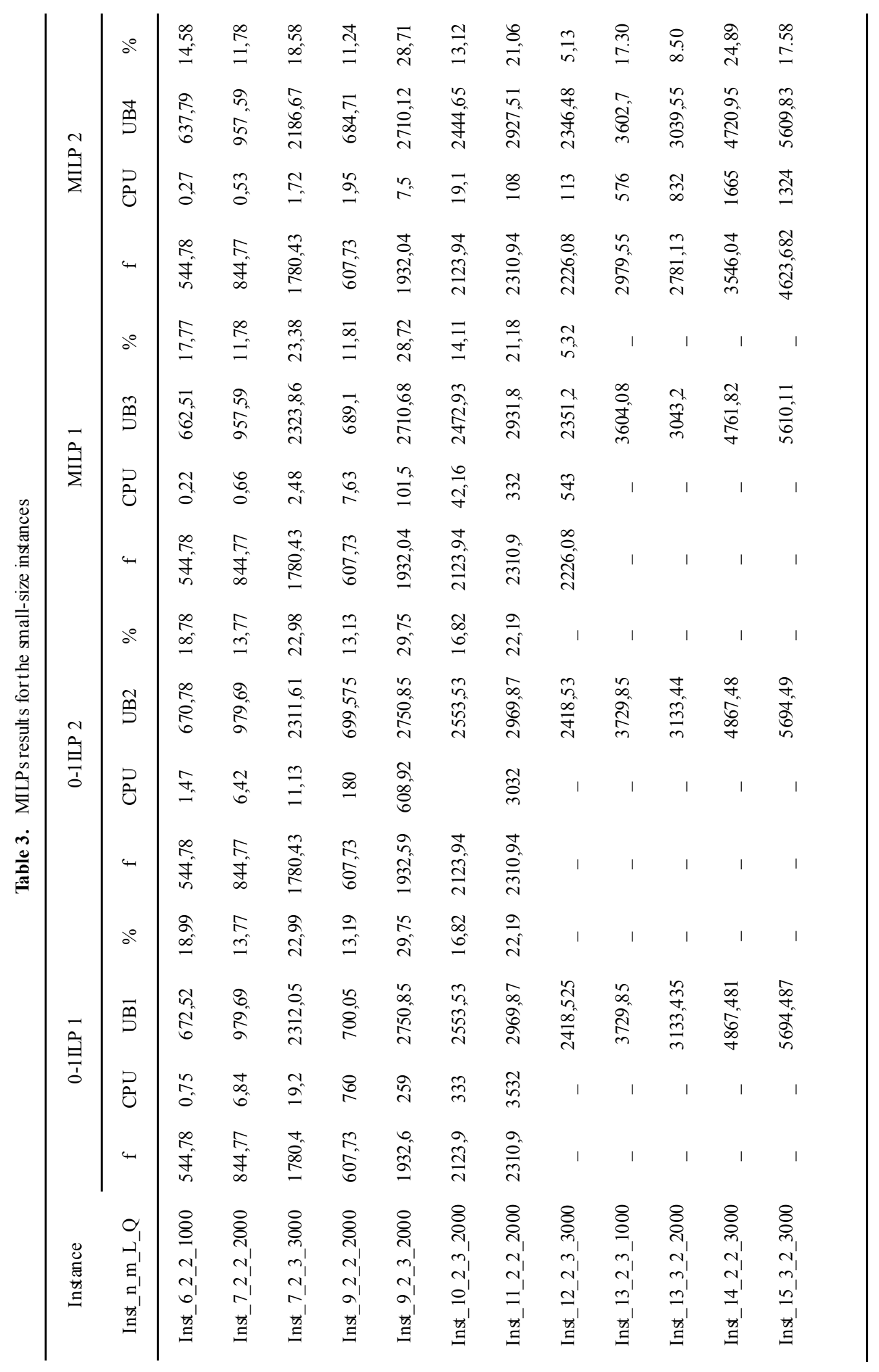


Table 4. Comparison between the Upper Bounds

\begin{tabular}{ccccc}
\hline Instance & $\left(\%^{*}\right) 1$ & $(\% *) 2$ & $(\% *) 3$ & $\left(\%^{*}\right) 4$ \\
\hline Inst_6_2_2_1000 & 0 & $-0,259$ & $-1,488$ & $-5,164$ \\
Inst_7_2_2_2000 & 0 & 0,000 & $-2,256$ & $-2,256$ \\
Inst_7_2_3_3000 & 0 & $-0,019$ & $-0,511$ & $-5,423$ \\
Inst_9_2_2_2000 & 0 & $-0,068$ & $-1,564$ & $-2,191$ \\
Inst_9_2_3_2000 & 0 & 0,000 & $-1,460$ & $-1,481$ \\
Inst_10_23_2000 & 0 & 0,000 & $-3,156$ & $-4,264$ \\
Inst_11_22_2000 & 0 & 0,000 & $-1,282$ & $-1,426$ \\
Inst_12_2333000 & 0 & 0,000 & $-2,784$ & $-2,979$ \\
Inst_13_2_3_1000 & 0 & 0,000 & $-3,372$ & $-3,409$ \\
Inst_13_322_2000 & 0 & 0,000 & $-2,880$ & $-2,996$ \\
Inst_14_22_3000 & 0 & 0,000 & $-2,171$ & $-3,010$ \\
& & & &
\end{tabular}

Table 5. The Performance of the heuristic procedures (small-size benchamark)

\begin{tabular}{|c|c|c|c|c|c|c|c|c|c|c|c|}
\hline $\begin{array}{c}\text { Instance } \\
\text { Inst } \mathrm{n} \mathrm{Q} \mathrm{m} \mathrm{D1} \mathrm{L}\end{array}$ & $\mathrm{f}$ & UB & H1 & $\mathrm{H} 2$ & $\mathrm{HCi}$ & $\mathrm{HCs}$ & $\mathrm{HCc}$ & VND & gap & gap* & gap** \\
\hline Inst_6_Q_1000_m_2_L2 & 544,78 & 637,79 & 533,07 & 533,07 & 533,071 & 544,787 & 544,787 & 544,787 & 0,00 & 0,15 & 0,02 \\
\hline Inst_7_Q 2000_m_2_L2 & 844,77 & 957,59 & 815,77 & 815,77 & 844,779 & 838,828 & 838,828 & 844,779 & 0,00 & 0,12 & 0,03 \\
\hline Inst_7_Q 3000_m_2_L3 & 1780,43 & 2186,67 & 1420,07 & 1420,07 & 1422,865 & 1460,897 & 1420,071 & 1460,99 & 0,18 & 0,33 & 0,03 \\
\hline Inst_9_Q 2000_m_2_L2 & 607,73 & 684,71 & 593,63 & 593,63 & 599,798 & 599,798 & 593,632 & 607,73 & 0,00 & 0,11 & 0,02 \\
\hline Inst_9_Q 2000_m_2_L3 & 1932,04 & 2710,12 & 1644,4 & 1473,24 & 1743,288 & 1696,424 & 1644,395 & 1932,04 & 0,00 & 0,29 & 0,15 \\
\hline Inst_10_Q 2000_m_2_L3 & 2123,94 & 2444,65 & 2064,21 & 1906,3 & 2075,956 & 2102,914 & 2064,241 & 2123,94 & 0,00 & 0,13 & 0,03 \\
\hline Inst_11_Q 2000_m_2_L2 & 2310,94 & 2927,51 & 1767,09 & 1775,67 & 1775,667 & 1767,088 & 1767,088 & 1775,67 & 0,23 & 0,39 & 0,00 \\
\hline Inst_12_Q 3000_m_2_L3 & 2226,08 & 2346,48 & 2210,68 & 2162,52 & 2217,934 & 2202,656 & 2150,677 & 2226,08 & 0,00 & 0,05 & 0,00 \\
\hline Inst_13_Q_1000_m_2_L3 & 2979,55 & 3602,70 & 2194,54 & 2194,54 & 2211,132 & 2242,851 & 2194,54 & 2424,46 & 0,33 & 0,33 & 0,09 \\
\hline Inst_13_Q 2000_m_3_L2 & 2781,13 & 3039,55 & 2623,57 & 2730,63 & 2660,211 & 2689,486 & 2526,086 & 2700,20 & 0,05 & 0,05 & 0,03 \\
\hline Inst_14_Q_3000_m_2_L2 & 3546,04 & 4720,95 & 3146,87 & 3141,51 & 3146,865 & 3149,766 & 3146,865 & 3149,77 & 0,11 & 0,33 & 0,00 \\
\hline Inst_15_Q_3000_m_3_L2 & 4623,682 & 5609,83 & 3935,41 & 3552,49 & 3935,414 & 3935,414 & 3960,382 & 4046,39 & 0,28 & 0,28 & 0,03 \\
\hline Inst_16_Q 3000_m_2_L3 & _ & 6060,13 & 2665,68 & 2678,24 & 2665,68 & 2665,68 & 2665,68 & 2678,24 & _- & 0,56 & 0,00 \\
\hline Inst_16_Q_3000_m_3_L3 & - & 5531,54 & 3220,31 & 3221,31 & 3305,529 & 3344,938 & 3232,182 & 3966,52 & - & 0,28 & 0,19 \\
\hline Inst_17_Q_2000_m_3_L2 & - & 3160,23 & 2711,65 & 2586,85 & 2740,154 & 2753,153 & 2598,295 & 2795,56 & - & 0,12 & 0,03 \\
\hline Inst_13_Q 2000_m_3_L2 & - & 6741,72 & 4236,92 & 4478,07 & 4236,919 & 4266,928 & 4266,928 & 4266,93 & - & 0,37 & 0,01 \\
\hline Inst_18_Q_3000_m_3_L3 & _- & 2686,73 & 3483,28 & 3473,86 & 3548,689 & 3573,86 & 3510,165 & 3777,69 & - & 0,03 & 0,08 \\
\hline Inst_19_Q_2000_m_3_L2 & - & 2686,73 & 2296,54 & 2318,93 & 2443,338 & 2367,516 & 2464,153 & 2511,30 & _- & 0,07 & 0,09 \\
\hline Inst_20_Q 3000_m_2 _L3 & - & 3258,2 & 2222,072 & 2255,93 & 2247,616 & 2298,96 & 2222,072 & 2533,85 & - & 0,22 & 0,12 \\
\hline Inst_20_Q 3000_m_2_L2 & - & 4347,68 & 2812,74 & 2812,74 & 2828,015 & 2846,575 & 2812,736 & 3063,55 & _ & 0,30 & 0,08 \\
\hline
\end{tabular}


Table 6. The Performance of the heuristic procedures (large-size benchamark)

\begin{tabular}{|c|c|c|c|c|c|c|c|c|c|c|c|}
\hline $\begin{array}{ll} & \text { Instance } \\
\text { CMT n Q m D1 L } \\
\end{array}$ & f & UB & H1 & $\mathrm{H} 2$ & HcI & $\mathrm{HcS}$ & $\mathrm{HcC}$ & VND & gap & gap* & gap** \\
\hline CMT_51_Q_160_m_1_D1_L2 & 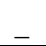 & 3113,45 & 2206,59 & 2206,59 & 2455,032 & 2277,637 & 2206,588 & 2842,89 & - & 0,09 & 0,22 \\
\hline CMT_51_Q_160_m_2_D1_L2 & - & 3736,64 & 2081,34 & 2183,8 & 2350,804 & 2130,816 & 2225,08 & 2461,25 & - & 0,34 & 0,15 \\
\hline CMT_51_Q_160_m_3_D1_L2 & - & 3748,72 & 2334,57 & 2334,57 & 2464,253 & 2371,068 & 2334,567 & 2487,91 & _- & 0,34 & 0,06 \\
\hline CMT_51_Q_160_m_4_D1_L2 & - & 3748,72 & 2230,98 & 2230,98 & 2343,928 & 2130,042 & 2099,984 & 2467,35 & - & 0,34 & 0,10 \\
\hline CMT_76_Q_140_m_1_D1_L2 & - & 3032,45 & 3524,05 & 2064,26 & 3769,024 & 3562,532 & 3524,05 & 4008,00 & - & 0,45 & 0,12 \\
\hline CMT_76_Q_140_m_2_D1_L2 & - & 5007,04 & 3498,02 & 3636,66 & 3879,421 & 3556,819 & 3612,512 & 4192,05 & - & 0,16 & 0,17 \\
\hline CMT_76_Q_140_m_3_D1_L2 & - & 6286,68 & 3397,44 & 3275,17 & 3646,122 & 3501,368 & 3398,561 & 4306,36 & _- & 0,32 & 0,21 \\
\hline CMT_76_Q_140_m_4_D1_L2 & - & 6731,73 & 3358,93 & 3395,48 & 3523,918 & 3427,507 & 3359,549 & 3740,66 & _- & 0,44 & 0,10 \\
\hline CMT_76_Q_140_m_5_D1_L2 & - & 6725,33 & 3325,81 & 3325,81 & 3328,465 & 3365,189 & 3325,814 & 3397,32 & - & 0,49 & 0,02 \\
\hline CMT_76_Q_140_m_6_D1_L2 & - & 6731,73 & 3443,2 & 3443,2 & 3350,861 & 3439,272 & 3366,666 & 3982,41 & - & 0,41 & 0,14 \\
\hline CMT_76_Q_140_m_7_D1_L2 & - & 6725,33 & 3310,97 & 3310,97 & 3315,43 & 3390,638 & 3327,282 & 3613,24 & _- & 0,46 & 0,08 \\
\hline CMT_101_Q_200_m_1_D1_L2 & - & 4058,84 & 3986,88 & 2748,67 & 4349,415 & 4047,658 & 3986,878 & 4558,84 & _- & 0,43 & 0,13 \\
\hline CMT_101_Q200_m_2_D1_L2 & - & 6422,85 & 3972,35 & 3981,45 & 4131,077 & 3938,675 & 3877,895 & 4457,71 & _- & 0,31 & 0,11 \\
\hline CMT_101_Q200_m_3_D1_L2 & - & 7393,38 & 3824,71 & 3849,6 & 4105,052 & 3922,299 & 3824,711 & 4494,23 & - & 0,39 & 0,15 \\
\hline CMT_101_Q_200_m_4_D1_L2 & - & 7386,04 & 3879,88 & 3879,88 & 4031,774 & 3871,932 & 3785,425 & 4189,23 & - & 0,43 & 0,07 \\
\hline CMT_101_Q_200_m_5_D1_L2 & - & 7386,04 & 3947,93 & 3947,93 & 3958,535 & 4003,192 & 3947,93 & 4463,13 & _- & 0,40 & 0,12 \\
\hline CMT_101_Q_200_m_6_D1_L2 & - & 7371,37 & 3556,48 & 3556,48 & 3560,052 & 3632,453 & 3560,146 & 4206,69 & - & 0,43 & 0,15 \\
\hline CMT_121_Q200_m_1_D1_L2 & - & 4395,66 & 3474,77 & 2869,42 & 5720,678 & 5612,154 & 5763,513 & 6280,78 & - & 0,40 & 0,45 \\
\hline CMT_121_Q_200_m_2_D1_L2 & - & 7360,11 & 5265,92 & 5390,88 & 5675,098 & 5428,476 & 5274,7 & 6563,07 & - & 0,11 & 0,20 \\
\hline CMT_121_Q200_m_3_D1_L2 & - & 9163,22 & 5218,69 & 5406,69 & 5620,678 & 5357,32 & 5222,098 & 7805,58 & - & 0,15 & 0,33 \\
\hline CMT_121_Q200_m_4_D1_L2 & - & 9753,08 & 5220,81 & 5187,36 & 5353,209 & 5176,06 & 5106,129 & 5687,89 & - & 0,42 & 0,08 \\
\hline CMT_121_Q_200_m_5_D1_L2 & - & 9745,53 & 5162,67 & 5352,12 & 5287,885 & 5303,714 & 5259,021 & 5625,18 & - & 0,42 & 0,08 \\
\hline CMT_151_Q_200_m_1_D1_L3 & - & 5235,16 & 5149,09 & 3073,91 & 5778,358 & 5380,735 & 5349,09 & 6310,27 & _- & 0,43 & 0,18 \\
\hline CMT_151_Q200_m_2_D1_L3 & - & 8216,37 & 4954,15 & 5451,05 & 5379,861 & 5086,381 & 4954,147 & 6328,29 & - & 0,23 & 0,22 \\
\hline CMT_151_Q_200_m_3_D1_L3 & - & 9839,11 & 5258,76 & 5098,55 & 5513,989 & 5369,196 & 5258,757 & 6203,90 & _- & 0,37 & 0,15 \\
\hline CMT_151_Q_200_m_4_D1_L3 & - & 10447,70 & 5332,26 & 5067,99 & 5605,328 & 5389,261 & 5334,541 & 5687,98 & _- & 0,46 & 0,06 \\
\hline CMT_151_Q_200_m_5_D1_L3 & - & 10447,70 & 5517,05 & 5517,05 & 5545,379 & 5569,375 & 5517,049 & 5602,45 & - & 0,46 & 0,02 \\
\hline CMT_151_Q200_m_6_D1_L3 & - & 10447,70 & 5530,36 & 5530,36 & 5545,207 & 5592,485 & 5529,918 & 5846,30 & - & 0,44 & 0,05 \\
\hline CMT_151_Q_200_m_7_D1_L3 & - & 10431,00 & 4851,51 & 4851,51 & 4857,404 & 4945,4 & 4863,401 & 5686,25 & _- & 0,45 & 0,15 \\
\hline CMT_151_Q200_m_8_D1_L3 & - & 10447,70 & 5034,29 & 5034,29 & 5028,23 & 5062,868 & 5024,861 & 5551,04 & - & 0,47 & 0,09 \\
\hline CMT_200_Q200_m_1_D1_L3 & - & 6292,79 & ,96 & 3273,25 & 7582,818 & 6852,94 & 6769,306 & 8062,51 & - & 0,46 & 0,52 \\
\hline CMT_200_Q200_m_2_D1_L3 & - & 10016,85 & 6760,24 & 6532,8 & 7562,591 & 6852,94 & 6760,458 & 7888,35 & - & 0,21 & 0,14 \\
\hline CMT_200_Q200_m_3_D1_L3 & - & 12033,30 & 6642,42 & 6889,04 & 7222,708 & 6728,453 & 6642,42 & 7340,18 & - & 0,39 & 0,10 \\
\hline CMT_200_Q_200_m_4_D1_L3 & - & 13348,75 & 6452,5 & 6894 & 7074,411 & 6643,446 & 6468,593 & 7898,69 & - & 0,41 & 0,18 \\
\hline CMT_200_Q200_m_5_D1_L3 & - & 14007,96 & 6367,01 & 6486,87 & 6899,801 & 6449,095 & 6367,011 & 7206,84 & - & 0,49 & 0,12 \\
\hline CMT_200_Q_200_m_6_D1_L3 & - & 14017,08 & 6707 & 6707 & 6976,519 & 6769,783 & 6706,998 & 7120,66 & - & 0,49 & 0,06 \\
\hline CMT_200_Q_200_m_7_D1_L3 & - & 14035,31 & 6983,25 & 6983,25 & 7092,305 & 7074,797 & 6985,065 & 7871,18 & 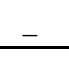 & 0,44 & 0,11 \\
\hline CMT_200_Q_200_m_8_D1_L3 & - & 14053,55 & 6659,92 & 6659,92 & 6957,153 & 6752,264 & 6683,254 & 7278,76 & - & 0,48 & 0,09 \\
\hline CMT_200_Q_200_m_9_D1_L3 & $\ldots$ & 14044,43 & 6370,01 & 6370,01 & 6763,057 & 6715,521 & 6661,164 & 7246,86 & _ & 0,48 & 0,12 \\
\hline CMT_200_Q200_m_10_D1_L3 & - & 14053,55 & 6069,91 & 6069,91 & 6209,274 & 6180,566 & 6094,38 & 7328,64 & - & 0,48 & 0,17 \\
\hline
\end{tabular}




\section{Conclusions}

In this paper, we describe a new variant of the vehicle routing problem namely the Profitable Vehicle Routing Problem with Multiple Trips. Two different strategies of sub-tours elimination constraints are used and for each strategy two different cases are defined. Thus, four mathematical models are obtained looking for optimal solutions. For large-size instances, two greedy constructive heuristics are proposed in a first solving step. Three Hill Climb ing algorithms based on three neighborhood structures are developed. With a VND procedure, these methods are managed to obtain improved solutions in a second solving step of the problem. The design of these methods is based on elements of reasoning to obtain intrinsically the best possible solutions from the first iterations. Enhanced diversification was covered by a broad research approach to effectively improve the results. Experimental study shows satisfactory results for small-size instances with MILPs using some cuts. Two strategies of sub-tours elimination constraints are used representing a good idea to formu late this kind of constraints The empirical results show the performance of the proposed constructive heuristics which provide quick solutions very close to the optimum, and also a satis factory enhancement by using the improvement procedures. In future work, with some adjustments and the introduction of well-studied perturbation moves, the obtained results could be further refined to ensure a better optimality of solutions.

\section{REFERENCES}

[1] N. Azi, M. Gendreau, J-Y. Potvin, "An exact algorithm for a single-vehicle routing problem with time windows and multiple routes" European Journal of Operational Research, vol.178 pp.755-766, 2007

[2] N. Azi, M. Gendreau, J-Y. Potvin, "An exact algorithm for a vehicle routing problem with time windows and multiple use of vehicles" European Journal of Operational Research, Vol. 202, no. 3, pp.756-763, 2010

[3] N. Azi, M. Gendreau, J-Y. Potvin, "An adaptive large neighborhood search for a vehicle routing problem with multiple trips" Technical report, CIRRELT, 2010-08 Montréal

[4] N. Azi, M. Gendreau, J-Y Potvin, "A dy namic vehicle routing problem with multiple delivery routes" Ann Oper Res DOI 10.1007/s 10479-011-0991-3

[5] R. Macedo, C. Alves, J.M. Valério de Carvalho, F. Clautiaux, S. Hanafi, "Solving the vehicle routing problem with time windows and multiple routes exactly using a pseudopolynomial model" European Journal of Operational Research, vol. 214, pp.536-545, 2011

[6] J. W. De Boer, "Approximate models and solution approaches for the vehicle routing problem with multiple use of vehicle and time windows" A thesis submitted to the graduate school of natural and applied sciences of middle east technical university (Jakarta), June 2008

[7] J. brandao, A. Mercer, "A tabu search algorithm for the multi trip vehicle routing and scheduling problem" European journal of Operational research, vol.100, pp. 180-191,1997

[8] U. Derigs, R. Kurowsky, U. Vogel, "Solving a real-world vehicle routing problem with multiple use of tractors and trailers and EU-regulations for drivers arising in air cargo road feeder services" European Journal of Operational Research, vol. 213 pp.309-319, 2011

[9] I. Gribkovskaia, B. O. Gullberg, K. J. Hovden, S. W. Wallace, "Optimization model for a livestock collection problem" International Journal of Physical Distribution \& Logistics Management, Vol. 36, no. 2, pp. 136-152, 2006

[10] C. Prins, "Efficient Heuristics for the Heterogeneous Fleet Multi trip VRP with Application to a Large-Scale Real Case" Journal of Mathematical Modelling and Algorithms, vol.1, pp. $135-150,2002$

[11] S-N. Sze, "A Study on the Multi-trip Vehicle Routing Problem with Time Windows and Meal Break Considerations" A thesis submitted in partial fulfillment of the requirements for the degree of Doctor of Philosophy University of Sydney, March 2011

[12] A. Kumar, R. Jagannathan, "Vehicle Routing with Cross Docks, Split Deliveries, and Multiple Use of Vehicles" A thesis Faculty of Auburn University, December 2011

[13] T. Grünert, H-J Sebastian, M. Thärigen, "The Design of a Letter-Mail Transportation Network by Intelligent Techniques" Proceedings of the 32nd Hawaii International Conference on System Sciences, 1999

[14] Y. Ren, M. Dessouky, F. Ordóñez, "The Multi-shift Vehicle Routing Problem with Overtime" Computers and Operations Research, Vol. 37, no. 11, pp. 1987-1998, 2010

[15] F. Hernandez, D. Feillet, R. Giroudeau, O. Naud, "Multi-trip vehicle routing problem with time windows for agricultural tasks", Fourth International Workshop on Freight Transportation and Logistics, France 2009

[16] M. Battarraa, M.Monacib, D.Vigo, "An adaptive guidance approach for the heuristic solution of a minimum multiple trip vehicle routing problem" Computers \& Operations Research, vol.36, pp. 3041-3050, 2009

[17] F. Hernandez, D. Feillet, R. Giroudeau, O. Naud, "A new exact algorithm to solve the Multi-trip vehicle routing problem with time windows and limited duration" lirmm00616667, version, Aug 2011

[18] Y. Yang, L. Tang, "A Filter-and-fan Approach to the Multi-trip Vehicle Routing Problem" Proceeding of the International Conference on Logistics Systems and Intelligent Management, vol. 3, pp. 1713-1717, 2010

[19] T-S. Chang, S-D. Wang, "Multi-Trip Vehicle Routing and Scheduling Problems" 40th International Conference on Computers and Industrial Engineering (CIE), 2010

[20] M. Campbell, M. Savelsbergh, "Efficient Insertion Heuristics for Vehicle Routing and Scheduling Problems" Ann Transportation Science, Vol. 38, no. 3, pp. 369-378, 2004

[21] A. Olivera, O. Viera, "Adaptive memory programming for the vehicle routing problem with multiple trips" Computers \& 
Operations Research, vol.34, pp. 28-47, 2007

[22] R.J. Petch, S. Salhi, "A multi-phase constructive heuristic for the vehicle routing problem with multiple trips" Discrete Applied Mathematics, vol.133, pp.69-92, 2004

[23] É-D. Taillard, G. Laporte, M. Gendreau, "Vehicle Routeing with Multiple Use of Vehicles" The Journal of the Operational Research Society, vol. 47, no. 8, pp. 1065-1070, 1996

[24] S. Salhi, R. J. Petch, "A GA Based Heuristic for the Vehicle Routing Problem with Multiple Trips" Journal of Mathematical Modeling and Algorithms, Vol.6, No 4, pp. $591-613,2007$

[25] C.K.Y. Lin a, R.C.W. Kwok, "Multi-objective metaheuristics for a location-routing problem with multiple use of vehicles on real data and simulated data" European Journal of Operational Research, vol.175, pp.1833-1849, 2006

[26] C. Huang, C-M. Lee, "A Study of Multi-Trip Vehicle Routing Problem and Distribution Center Location Problem" POMS 22nd Annual Conference Reno, Nevada, U.S.A. April 29 to May 02, 2011

[27] F. Alonso, MJ Alvarez, JE Beasley, "A tabu search algorithm for the periodic vehicle routing problem with multiple vehicle trips and accessibility restrictions" Journal of the Operational Research Society, vol.59,pp.963-976, 2008

[28] B. Crevier, J-F. Cordeau, G. Laporte, "The multi-dep ot vehicle routing problem with inter-depot routes" European Journal of Operational Research, vol.176, pp.756-773,2007

[29] JCS. Brandao and A. Mercer, "The Multi-Trip Vehicle Routing Problem" The Journal of the Operational Research Society, Vol. 49, no. 8, pp. 799- 805,1998

[30] B-L. Golden, G. Laporte, E-D. Taillard, "An adaptative memory heuristic for a class of vehicle routing problems with minmax objective" Computers operational research, vol 24, no 5, pp 445-452, 1997

[31] C. Koc, I. Karaoglan, "A branch and cut algorithm for the vehicle routing problem with multiple use of vehicles" Proceedings of the 41st International Conference on Computers \& Industrial Engineering, Los Angeles, 2011

[32] "The Vehicle Routing Problem with Multiple Trips" Available on http://citeseerx. ist.psu.edu/viewdoc/summary? doi $=10.1 .1 .84 .3651$

[33] S. kagaya, S. Kikuchi, R. A. Donnelly, "Use of fuzzy theory technique for grouping of trips in the vehicle routing and scheduling problem" European Journal of Operational
Research vol.76, pp.143-154, 1994

[34] D. Feillet, P. Dejax, M. Gendreau, "Traveling salesman problems with profits" Transportation Science, vol.39, no. 2, pp 188-205, 2005

[35] A. Dell, F. Maffioli, P. Vanbrand, "On prize collecting tours and the asymmetric travelling salesman problem" International transaction operational research, vol. 2, no.3, pp 29-308, 1995

[36] C. Archetti, D. Feillet, A. Hertz and M.G. Speranza, "The capacitated team orienteering and profitable tour problems" Journal of the Operational Research Society, vol. 60, pp. 831842,2009

[37] C. Archetti, N. Bianchessi , M.G. Speranza, "Optimal solutions for routing problems with profits" Discrete Applied Mathematics, Available online 14 January 2012

[38] N. Aras, D. Aksen, M.T. Tekin, "Selective multi-dep ot vehicle routing problem with pricing" Transportation Research, vol.19, no. 5, pp. 866-884, 2011

[39] E. Thorson, J. Holguín-Veras, J.E. Mitchell, "Multi vehicle routing with profits and market competition", available on http ://eaton.math.rpi.edu/faculty/Mitchell/papers/VRPcompe tition.pdf

[40] M. Andres, F. Hani, S. Mahmassani, P. Jaillet, "Pricing in Dynamic Vehicle Routing Problems" Transportation Science, vol. 41, no. 3, pp. 302-318, 2007

[41] C. Archetti, D. Feillet, A. Hertz, M. G. Speranza "The undirected capacitated arc routing problem with profits" Computers \& Operations Research, Vol. 37, no. 11, pp. 1860-1869, 2010

[42] E.E. Zachariadis, C.T. Kiranoudis, "Local search for the undirected capacitated arc routing problem with profits" European Journal of Operational Research, vol. 210, no. 2, pp. $358-367,2011$

[43] P. Vansteenwegen W. Souffriau, D. V. Oudheusden, "The orienteering problem: A survey" European Journal of Operational Research, vol. 209, no. 1, pp. 1-10, 2011

[44] C.E. Miller, A.W. Tucker, R.A. Zelim, "Integer programming formulations and traveling salesman problem" Journal of association for Computing Machinery, vol. 7, pp. 326-329, 1960

[45] I. Kara, G. Laporte, T. Bektas, "A note on the lifted MillerTucker-Zemlin subtour elimination constraints for the capacitated vehicle routing problem" European Journal of Operational Research, vol. 158, pp.793-795, 2004 